\title{
COMMERCIAL OFF THE SHELF GROUND CONTROL SUPPORTS CALIBRATION AND CONFLATION FROM GROUND TO SPACE BASED SENSORS
}

\author{
M. Danielová ${ }^{\mathrm{a}}$ P. Hummel ${ }^{\mathrm{b}}$ \\ a AccuEarth s.r.o., Sokolovská 49, Praha 8, Czech Republic - mariana@accuearth.eu \\ ${ }^{\mathrm{b}}$ CompassData, Inc, Centennial, Colorado, United States of America - phummel@ compassdatainc.com
}

KEY WORDS: GCPs, Ground Control Points, Photo Identifiable Ground Control, Remote Sensed Ground Control Points, RSGCPs ${ }^{\circledR}$

\begin{abstract}
:
The need for rapid deployment of aerial and satellite imagery in support of GIS and engineering integration projects require new sources of geodetic control to ensure the accuracy for geospatial projects. In the past, teams of surveyors would need to deploy to project areas to provide targeted or photo identifiable points that are used to provide data for orthorecificaion, QA/QC and calibration for multi-platform sensors. The challenge of integrating street view, UAS, airborne and Space based sensors to produce the common operational picture requires control to tie multiple sources together. Today commercial off the shelf delivery of existing photo identifiable control is increasing the speed of deployment of this data without having to revisit sites over and over again. The presentation will discuss the processes developed by CompassData to build a global library of 40,000 control points available today. International Organization for Standardization (ISO) based processes and initiatives ensure consistent quality of survey data, photo identifiable features selected and meta data to support photogrammetrist, engineers and GIS professionals to quickly deliver projects with better accuracy.
\end{abstract}

\section{INTRODUCTION}

The idea of creating a global library of control points available off the shelf appeared based on the increasing requests for ground control points located all over the world. The individual request of the points had to be fulfilled by deploying team of surveyors into the desired area to conduct the geodetic measurement of targeted or photo identifiable points by GNSS methods, like GPS, Glonass, Galileo, etc.

The evolution in the aerial and satellite imagery field is rapidly increasing and it is possible to develop products with higher resolution and accuracy. Better sensors also cover more land area with lower costs and thus there is larger demand for updated imagery products with higher resolution and improved accuracy. This usually affects the distribution and density of Ground Control Points (GCPs) and surveyors might have to revisit formerly covered areas.

Avoiding revisits in the long term was the main idea to start a global GCP archive. A huge advantage, besides avoided travel costs, is the availability of GCPs before the acquisition of the imagery. The archive is accessible online and users select points in different stages of a project.

Obviously to maintain the usefulness of the archived points features are chosen wisely for different purposes and permanency. Targeted points are generally not as useful as for other projects than to which they were intended to mostly because of their short life spans. From this follows that photo identifiable points are preferred solution to maintain the suitability of the entire archive (Rees, 1999). Selection of the photo identifiable points is discussed in more details in section 3.3.

\section{REASON TO BUILD A GLOBAL ARCHIVE OF GROUND CONTROL POINTS}

The idea leading CompassData to develop the archive of ground control points originated from the needs and habits of the GIS community. One challenge for the remote sensing profession is an accurate, consistent, and cost effective source of GCPs. Professionals see GCPs as an expensive and time consuming necessity for image production. These issues result in these professionals doing workarounds or accept a less accurate and consistent end product to avoid the cost and hassle of getting good ground truth data. Using multiple survey companies for large scale projects requires the imagery producer to manage multiple vendors, with different survey equipment and variable processing methods that introduce additional factors that could negatively affect the accuracy of the delivery, delay time lines, and add to the overhead costs.

The above issues had been identified many years ago and CompassData had the vision to develop a business model to collect consistent and geodetically accurate data that are collected once and licensed many times to enable professionals to produce the best quality product, quickly at a reasonable cost. Managing one provider, who provides consistent products over large geographic areas, reduces the potential for errors in the production of the end product. This factory approach to data delivery reduces the cost of production for remote sensing data providers versus managing individual deliveries from many different surveyors.

\subsection{Methods of Collections}

The global archive consists of ground control points collected by two methods. Most of the points are collected by state of the art GNSS technology in order to ensure the required accuracy. However, some points are in areas which are very difficult to access due to military restrictions, political tensions, or simply high costs. In these cases, points are derived from other remotely sensed data. In order to distinguish Ground Control Points (GCPs) measured by common geodetic approaches, the term remote sensed ground control points (RSGCPs ${ }^{\circledR}$ ) is introduced (Hummel, 2011). The main advantages and disadvantages of these two methods are described in the two following subsections. 
2.1.1 Classical geodetic approaches: The standard and classic approach to survey GCPs is with GNSS equipment. It is the most flexible method since the surveyor can chose exactly the feature in the field he surveys. Often multiple features are available whereof the surveyor makes his best choice based of experience. Also a customer selected features of the imagery and the surveyor is best equipped in the field to interpret this choice. The calculation of the coordinates follows standard GNSS processing practices. Here it should be noted that challenges exist in the establishment of the correct geodetic datum that might or might not be effected by tectonic displacements and its realization of the geodetic datum that potentially differs by multiple meters. Differences are seen between datum definitions that are often decades old. Another main advantage of a survey with GNSS equipment is the achievable centimeter accuracy with the right efforts. However, most applications require lower accuracies.

Nevertheless, it is important to mention the main disadvantage of these methods in comparison with an RSGCP ${ }^{\circledR}$ solution: A survey team has to physically reach the location. There are many places around the world where it is impossible to travel for many reasons (security, denied areas, high costs). One way to safely get control points from these areas is to derived the points using TerraSAR-X.

2.1.2 Remote sensed ground control points: The Airbus Defense and Space department in charge of the TerraSAR-X satellite can produce RSGCPs ${ }^{\circledR}$ with an unrivalled accuracy. Features have to be visible in the Radar imagery and in the optical imagery. If manmade objects, like street lights, power-poles, or cell-antennas are available in the area of interest, the coordinates can be provided in each of the cardinal directions with an accuracy better than $1 \mathrm{~m}$. Traditional, terrestrial based survey is still the ideal source for control, but the usability of RSGCPs $®$ in inaccessible or denied access areas around the globe has a great advantage. (Hummel, 2011)

\subsection{Challenges}

There are challenges ranging from the obvious logistical deployment to the final processing of the data, as well as the availability of clearly defined features at the site. The availability challenge is frequently the toughest one. In the most parts of the developed world the infrastructure of the road network provides concrete and asphalt corners, white stripes, manholes and so forth while in other parts of the world the road network consists of gravel roads without such preferred features. Below is a nice flowerbed with clear geometry that is ideal as a photo-identifiable feature for satellite imagery.

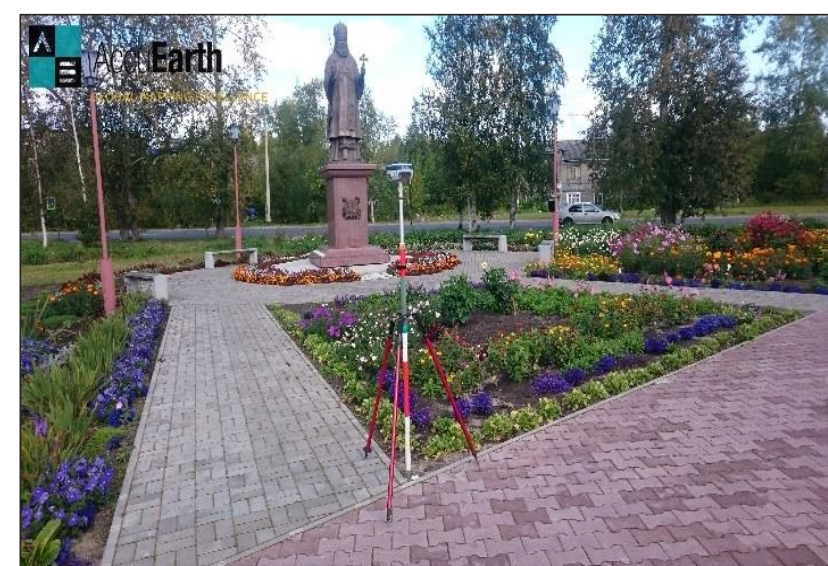

Figure 1. Clear Photo-identifiable GCP
A rare concrete corner that still doesn't provide a clear geometry due to the raised elevation, the ornamental slope, vicinity of trees, and the lack of contrast - nevertheless one of the best features at this given location.

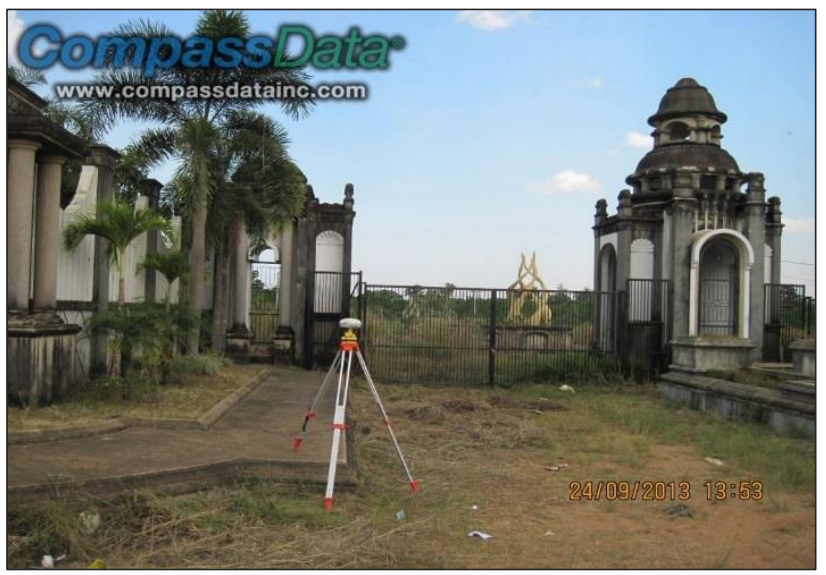

Figure 2. Difficult Photo-identifiable GCP

As the challenges for finding good photo-identifiable features in certain geographic regions rise, usually the challenge of processing GNSS data due to lack of continuously operating base stations and less advanced geodetic datum systems also rises.

\section{QUALITY ASSURANCE}

\subsection{ISO certification}

As global provider of GCPs CompassData works with a customer base that is operating globally, too. International applications generally have more obstacles than local applications and a smooth workflow of consistent data and consistent quality is desired. CompassData has decades of experience with hundreds of projects around the world. Starting in 2013 this knowhow was assembled with the goal to create guidelines to ensure consistent data. From simple steps to the complex determination of geodetic datum systems, ISO 9001:2008 certified QA and QC processes have been established.

Constant improvement and client satisfaction are monitored successfully. In addition to the ISO certification, a DO-200A certification by the US Federal Aviation Authority, with stringent requirements for aviation products has also been implemented.

\subsection{Selection of Ground Control Points}

Each ground control point is represented by different man-made features. Selecting suitable GCPs in the field which is also visible on low resolution imagery can be difficult. (Janssen, 2009) There are several aspects that should be accomplished to ensure the suitability of the point's features for orthorectification of aerial or satellite imagery, sensor calibration, etc. 
3.2.1 Contrast: The contrast between the features representing the ground control point and its surrounding must be evident. The characteristics of the feature's materials and its surroundings have to be considered as well since some materials can change contrast over time. For example, it may appear that a corner of new asphalt patch on a road is a great point at the time, however dark black asphalt color is usually fades quickly and it loses its contrast with the surrounding road, or it is likely to happen that a field with no vegetation during winter can be fully vegetated during spring and summer.

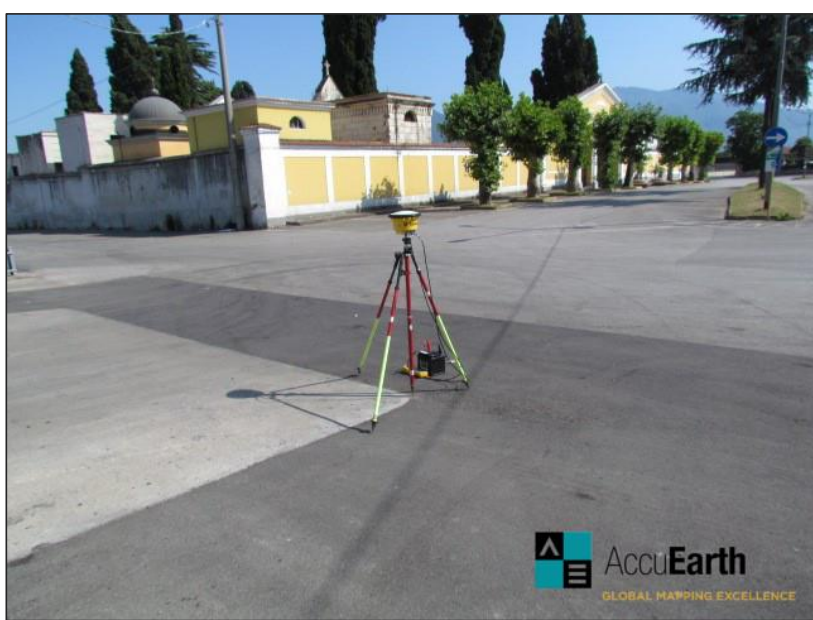

Figure 3. Asphalt and Concrete - different shades of Grey

Moreover, it is important to take into account that the ground control points can be used on black and white imagery. In cases like this choosing point with appropriate contrast is even more important.

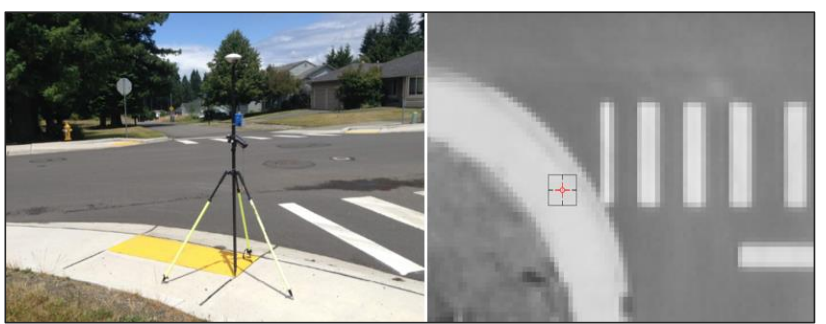

Figure 4. Contrast of a yellow feature is undetectable in the black and white imagery

3.2.2 Dimension: There can be a feature with great contrast but still not suitable for processes of orthorectification or calibration. The dimensions of the point's feature have to be bigger that the resolution of the imagery used in further applications. The majority of the points in the archive was collected using the rule of thumb that the feature must be at least 2 times, ideally 3 times bigger that the resolution of the imagery.

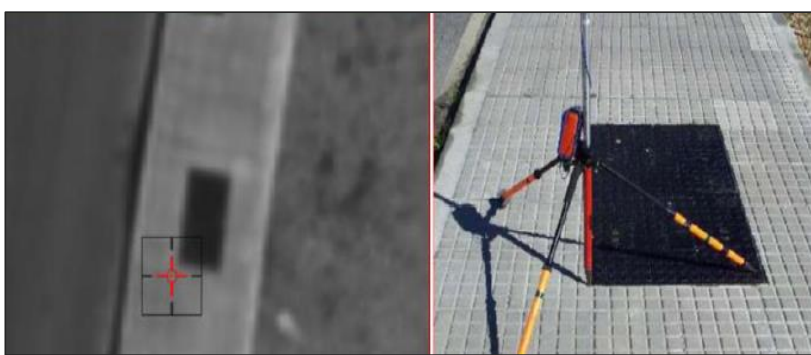

Figure 5. This black vault is visible on high resolution imagery, but will blend in with lower resolution imagery by satellites.
3.2.3 Sharp edges: Standard GNSS processing achieves a horizontal accuracy far better than $10 \mathrm{~cm}$. In order to make sure that this horizontal accuracy can be fully utilized, the feature collected has to be clearly identifiable on the imagery, not only regarding its contrast but the sharpness of the feature edges. The sharper the edges, the easier it is to determine the position on an image. Painted features often deteriorate quicker than sharp hard features such as sidewalk corners.

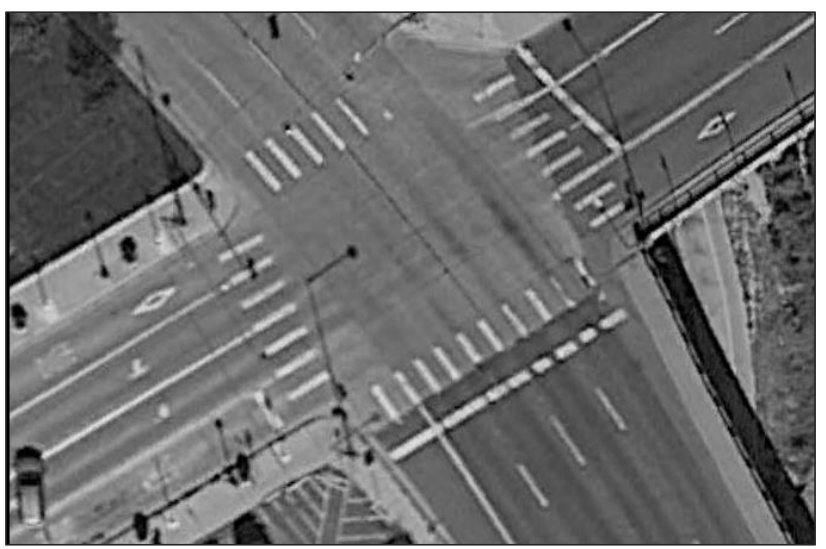

Figure 6. In this aerial image the white stripes seem to be clearly defined, while the visit in the field revealed that the corners were faded and not usable.

3.2.4 Elevated features: The ideal feature has the same elevation as its surrounding, i.e. it is not an elevated feature. However, ground control points are measured in real world. When there is a lack of flat man-made structures, corners of elevated features can supply good control. Nevertheless, each height of elevated feature, even the height of small curbs, should be noted in the point's metadata.

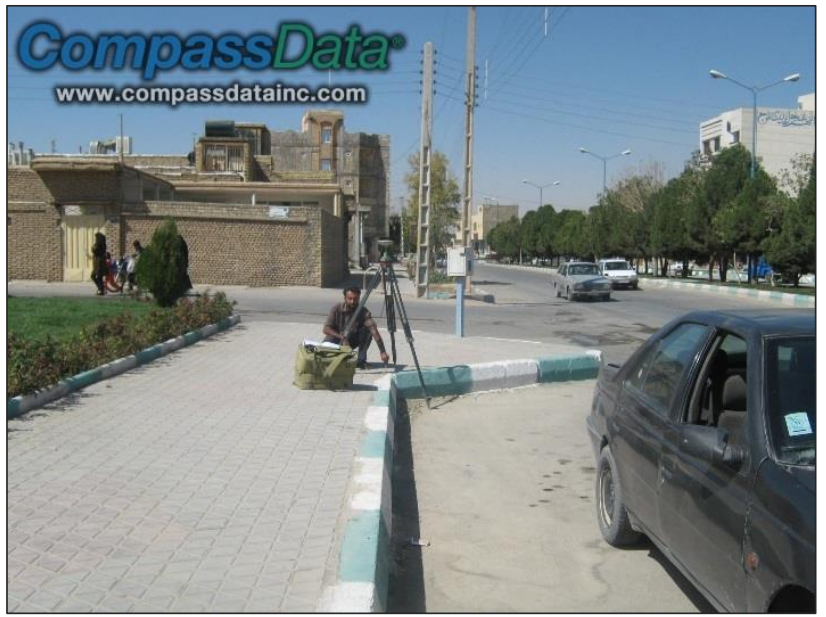

Figure 7. Elevated Curb as GCP 
3.2.5 Requirements for LiDAR point clouds: LiDAR point cloud data also requires GCPs. Obviously points used for LiDAR products have different requirements. Feature identifiability does not depend on the color contrast. Often it is not even required to identify the horizontal location and solely the vertical component is important. It is distinguished between data controlling points and testing points. Both types are in the open and unobstructed from any vegetation, but used differently in the process either as a control point or a test point to quantify the quality. Also there are points that are purposely surveyed under vegetation to test the vegetation removal process.

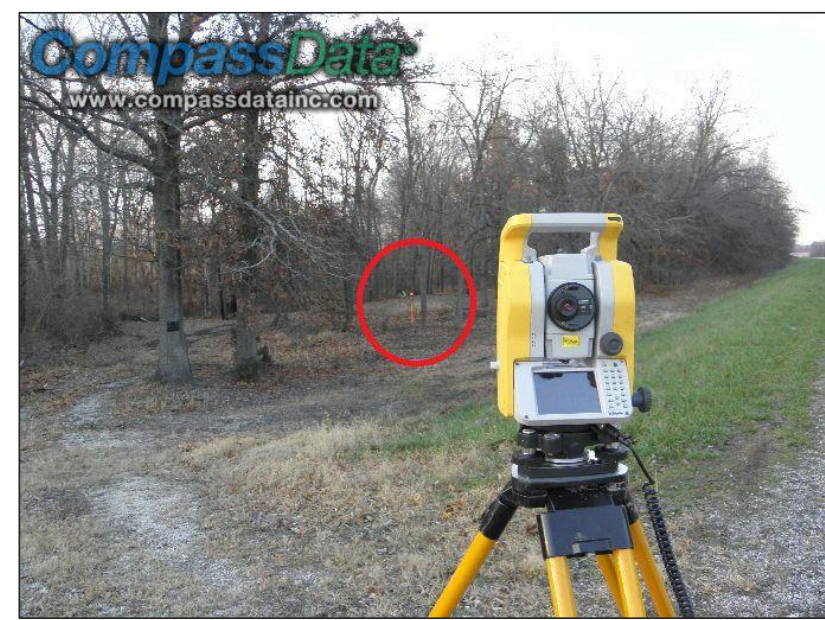

Figure 8 . Total station and surveyed test point under vegetation in red circle

\subsection{Metadata}

Having appropriate metadata is necessary to help photogrammetrist, engineers and GIS professionals to quickly deliver projects with better accuracy. Thus each point from the global archive is accompanied with photos from different orientation showing the collected feature and its surroundings; station diagram containing drawing of the surrounding and short description of the area including additional information like height of the feature if it is elevated, names of streets, etc.; and accuracy report showing the results of the processing and stating the final accuracy of the coordinates. Finally, all coordinates are based on one of the many geodetic datum systems, often just referred to as coordinate system with a projection and heights above ellipsoid and orthometric heights. The appropriate metadata is crucial for any application.

\section{CONCLUSION}

GCPs and RSGCPs ${ }^{\circledR}$ provide a crucial geometric ground truth to any geospatial product and especially any kind of geospatial imagery. GCPs have a higher accuracy than the imagery and a visibility to match the resolution of the imagery. As new sensors become better and cover more land area, the greater the need for good GCPs that control the ground truth with an appropriate distribution.

Each geospatial product has inherited a geometric quality throughout their creation. Overlaying independent GCPs is a solid method to assess this quality.

The global archive of ground control points (see Figure 10) poses a huge advantage in consistency and accuracy of delivered world wide data. This allows remote sensing professionals to rely on a single GCP provider even in project covering large geographic areas and thus avoid situation of using GCPs from multiple sources which can result in inconsistent product or errors in computation.

Figure 9 and 10 illustrate the growing demand for GCPs and locates areas of demand for high end geospatial products.

Figure 9. Global archive of ground control points

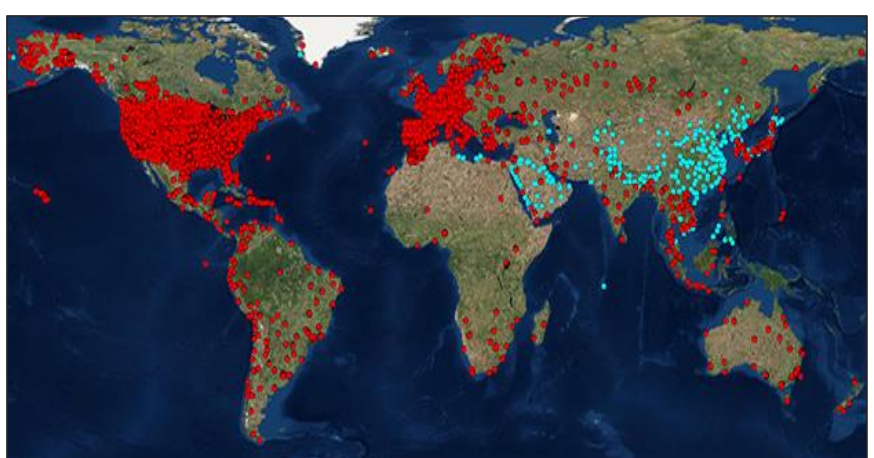

Figure 10. Global archive of GCP (red) and RSGCPs ${ }^{\circledR}$ (cyan)

\section{REFERENCES}

ASPRS, 2004. 15.1.2.6 Panning for Ground Control, Manual of Photogrammetry, Fifth Edition. ASPRS Bethesda, Maryland, USA, pp. $1111-1115$.

Hummel, P., 2011. Remotely Sensed Ground Control Points, Denver, Colorado, USA http://www.compassdatainc.com/wpcontent/uploads/TIM.pdf (1 April 2016).

Janssen, L.L. ed., 2009. Principles of remote sensing. ITC, International Institute for Aerospace Survey and Earth Sciences. ITC, Enschede, Netherlands, pp. 229-231.

Kraus, K., 2007. Photogrammetry, Second Edition. De Gruyter, Berlin, Germany, pp. $219-228$.

Rees, G., 1999. The remote sensing data book. Cambridge university press, pp. 74-89. 